\title{
Fabrication and Properties of High-Content Keratin/Poly (Ethylene Oxide) Blend Nanofibers Using Two-Step Cross-Linking Process
}

\author{
Yong Liu, ${ }^{1}$ Xia Yu, ${ }^{1}$ Jia Li, ${ }^{1}$ Jie Fan, ${ }^{1,2}$ Meng Wang, ${ }^{3}$ Tong-Da Lei, ${ }^{1}$ \\ Jian Liu, ${ }^{4}$ and Dongwei Huang ${ }^{5}$ \\ ${ }^{1}$ Key Laboratory of Advanced Textile Composites, Ministry of Education, School of Textiles, Tianjin Polytechnic University, \\ 399 West Binshui Road, Tianjin 300387, China \\ ${ }^{2}$ National Engineering Laboratory for Modern Silk, College of Textile and Clothing Engineering, Soochow University, \\ Suzhou 215123, China \\ ${ }^{3}$ Tianjin Medical University Cancer Institute and Hospital, Huanhuxi Road, Hexi District, Tianjin 300060, China \\ ${ }^{4}$ Department of Textiles, Zhejiang Fashion Institute of Technology, 495 Fenghua Road, Ningbo, Zhejiang Province 31521, China \\ ${ }^{5}$ School of Science, Tianjin Polytechnic University, 399 West Binshui Road, Tianjin 300387, China
}

Correspondence should be addressed to Jie Fan; fanjie@tjpu.edu.cn and Meng Wang; wm@medmail.com.cn

Received 26 October 2014; Revised 6 January 2015; Accepted 6 January 2015

Academic Editor: Yuqin Wan

Copyright (C) 2015 Yong Liu et al. This is an open access article distributed under the Creative Commons Attribution License, which permits unrestricted use, distribution, and reproduction in any medium, provided the original work is properly cited.

\begin{abstract}
High-content keratin/poly (ethylene oxide) (PEO) (90/10) blend nanofibers were prepared by electrospinning combined with a two-step cross-linking process. The keratin/PEO aqueous solution was firstly mixed with ethylene glycol diglycidyl ether (EGDE) as cross-linker and then electrospun into nanofibers. The resulting nanofibrous mats were cross-linked with EGDE vapor to decrease the solubility of nanofibers in water. The morphologies and properties of electrospun fibers were investigated by SEM, FTIR, TG, $\mathrm{XRD}$, and contact angle testing, respectively. The results showed that the morphologies of nanofibers were uniform at the fiber average diameter of $300 \mathrm{~nm}$ with negligible bead defects by adding EGDE to keratin/PEO solutions. The cross-linking results showed that EGDE vapor could improve the hydrophobic property of blended nanofibers. The crystallinity of the keratin/PEO blend nanofiber mat increased from $13.14 \%$ for the uncross-linked sample to $21.54 \%$ and $35.15 \%$ for the first cross-linked and second cross-linked samples, respectively. Free defect nanofiber mats with high keratin content producing from this two-step cross-linking process are particularly promising for tissue engineering and cell-seeded scaffold.
\end{abstract}

\section{Introduction}

Keratin, as the major protein component of hair, wool, feathers, nails, and horns, belongs to a family of fibrous structural proteins and can be used in a variety of biomedical applications due to its biocompatibility and biodegradability [1-7]. Among these sources, human hair keratin emerges as an attractive protein because it can be easily obtained from the unlimited supply of human hair, making it abundant and readily available [8]. Electrospun nanofiber mats have many potential biomedical applications for their attractive features such as high surface-to volume ratio and very high porosity [9-11]. In addition, the structure and fiber distribution of the mats can be controlled to provide them with necessary mechanical property and induction during the cell culture process [12]. Due to their excellent properties, a handful of research groups have tried to create keratin fibrous matrices through electrospinning technology [1316]. However, because of the low molecular weight and low viscosity of extracted keratin, most researchers have to resort to fabricate nanofibrous mats using keratin in combination with other natural or synthetic polymers [17].

Poly (ethylene oxide) (PEO) is a water-soluble polymer with good biocompatibility, low toxicity, and excellent spinnability. It has been proved that the fiber-forming property of keratin solution can be greatly improved by proper 
addition of PEO [13, 18]. When PEO is blended with keratin in ratio of 30/70, the viscosity of spinning solutions increased and could be electrospun into continuous nanofibers with few defects. However, when the keratin content is further increased, the bead-like defects would appear on the fiber. How to prepare keratin/PEO nanofiber mat with high keratin content is the urgent problem to be solved for medical applications such as tissue engineering. Xin et al. [19] proposed a method to prepare keratin/PEO nanofiber mat with high keratin content. In their method, keratin was chemically modified by reacting sulfide side group with iodoacetic acid to enhance its solubility in organic solvent. Then, the modified keratin was blended with PEO in weight ratio of 90/10 and dissolved in 2, 2, 2,-trifluoroethanol (TFE) for preparation of nanofibers. Although organic solvent can increase keratin content in the resulting samples, the residual solvent in the nanofibers may cause an unexpected risk such as allergy. In addition, comparing with water as spinning solvent, organic reagent as solvent is not environmental friendly and not economic. Another problem to be solved for making biomedical keratin/PEO blend nanofiber mat is that the blend nanofiber mats are easily to be dissolved in water because of the water solubility of keratin and PEO. Xin et al. [19] also suggested that the water tolerance of the keratin/PEO blend nanofiber mat should be improved by cross-link treatment.

Ethylene glycol diglycidyl ether (EGDE) is a diepoxy cross-linker with two epoxide groups on either end. The epoxide group is reactive cyclic ether composed of three ring atoms, which make EGDE easily react with amino group on the amino acid at the end or in the middle of the keratin molecules in neutral condition; see Figure 1. In this condition, the keratin molecules can be extended by EGDE. Therefore, the spinnability of keratin and its content in the keratin/PEO blend nanofiber mat might be improved. Furthermore, EGDE might also provide an appropriate choice for improving the waterproof performance of the blend nanofiber mat.

In this work, a high keratin content keratin/PEO (90/10) blend nanofiber mat was fabricated by electrospinning using aqueous solution after cross-linking treatment with ethylene glycol diglycidyl ether (EGDE). In order to improve the water resistance of the nanofiber mat, the obtained blend nanofiber mat was further treated with EGDE vapor. The properties of nanofibers were investigated by SEM, FTIR, TG, and a video optics contact angle apparatus.

\section{Experimental}

2.1. Extraction of Human Hair Keratin. Human hair was obtained randomly from a local hair salon, washed extensively, rinsed with petroleum ether, and then dried at room temperature for $24 \mathrm{~h}$ and cut into $5 \mathrm{~mm}$ length. The extraction of keratin from human hair was performed according to the previously reported method [20]. The human hair (6g) was shaken in $200 \mathrm{~mL}$ of aqueous solution containing $7 \mathrm{M}$ urea, $2 \mathrm{wt} \% \mathrm{SDS}$, and $5 \mathrm{wt} \% \mathrm{Na}_{2} \mathrm{~S}_{2} \mathrm{O}_{5}$ at $95^{\circ} \mathrm{C}$ for $4 \mathrm{~h}$. The resulting mixture was filtered through a stainless-steel mesh, and subsequently dialyzed against $3 \mathrm{~L}$ of distilled water in

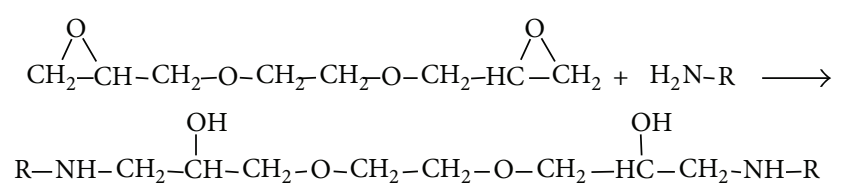

FIGURE 1: The schematic reaction of keratin with EGDE in neutral condition.

cellulose tubing (molecular weight cut off $=8000-14000 \mathrm{Da}$ ) for $36 \mathrm{~h}$ with three changes of $\mathrm{H}_{2} \mathrm{O}$. Finally, the keratin solution was then lyophilized to obtain keratin powder.

2.2. First Cross-Linking in Keratin/PEO Solutions. PEO (molecular weight $=400000 \mathrm{Da})$ power $(10.5 \mathrm{~g})$ was stirred in $150 \mathrm{~mL}$ of distilled water at $45^{\circ} \mathrm{C}$ for about $4 \mathrm{~h}$. Keratin powder $(0.126 \mathrm{~g})$ was first dissolved in $7 \mathrm{wt} \%$ PEO solution $(0.2 \mathrm{~mL})$ and then distilled water was added into the mixture. The final mixture solution was $2 \mathrm{~mL}$. Subsequently, EGDE $(0.125 \mathrm{~mL})$ was mixed into the keratin/PEO solution for electrospinning. The mixtures were stirred for $30 \mathrm{~min}$ at $60^{\circ} \mathrm{C}$ to ensure complete dissolution.

\subsection{Fabrication of Electrospun Keratin/PEO Nanofibrous} Mats. After dispensing the keratin/PEO solution after crosslinking by EGDE, electrospinning was performed at a constant feed rate of $0.5 \mathrm{~mL} / \mathrm{h}$, an accelerating voltage of $16 \mathrm{kv}$ and the distance of $15 \mathrm{~cm}$ from the syringe tip to collector for ensuring complete evaporation of solvent. The nanofibrous mats were produced with a deposition time of $30 \mathrm{~min}$.

2.4. Second Cross-Linking of Keratin/PEO Nanofibrous Mats. In order to increase the water resistance of nanofibers for their biomedical applications, keratin/PEO nanofiber mat was further cross-linked with EGDE vapor $(99.5 \%)$ at $80^{\circ} \mathrm{C}$ for $5 \mathrm{~h}$.

2.5. Characterization. The morphologies of the samples were analyzed using a scanning electron microscope (SEM, TM-1000, Hitachi, Japan). A Fourier transform infrared spectroscopy (FTIR, TENSOR37, Bruker, Germany) was employed to determine the chemical composition of the untreated keratin/PEO nanofibers, first cross-linking keratin/PEO nanofibers and second cross-linking keratin/PEO nanofibers. Infrared absorbance spectra of the samples were recorded at wavelengths $400-4000 \mathrm{~cm}^{-1}$.

A video optics contact angle apparatus (JY-80, Chende Dingsheng Test machine equipment Co., Ltd., China) was used to assess the hydrophilicity of keratin/PEO nanofibers obtained in these experiments.

\section{Results and Discussion}

3.1. Morphologies of Nanofiber Mat. Figure 2 shows the SEM micrographs of the nanofiber mats produced from spinning solution containing $90 \mathrm{wt} \%$ keratin. As shown in Figure 2(a), there were bead defects in nanofibers when the spinning solution had not recieved EGDE cross-linking pretreatment. The 


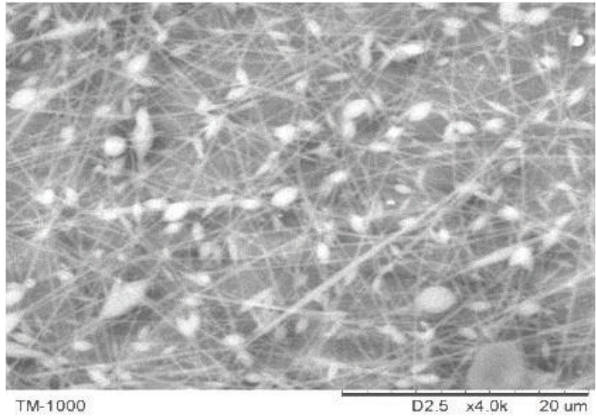

(a) Untreated keratin/PEO nanofibers mat

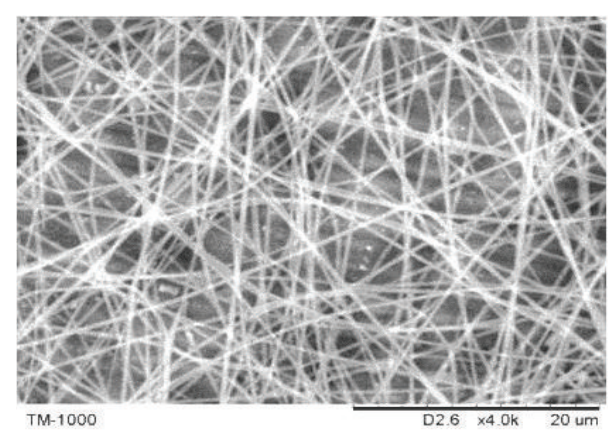

(b) First cross-linking keratin/PEO nanofibrous mat

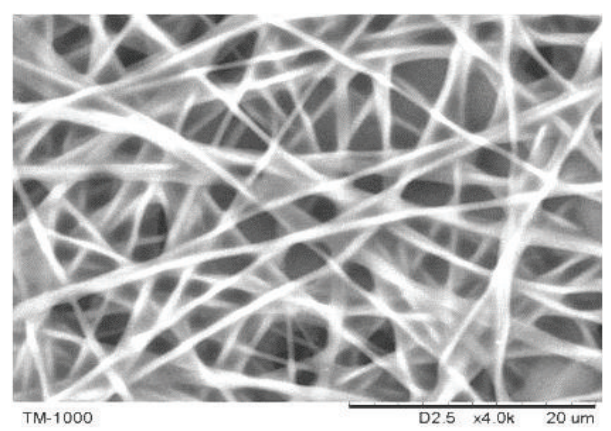

(c) Secondary cross-linking keratin/PEO nanofibrous mat

FIGURE 2: Scanning electron micrographs of nanofiber mats (a) uncross-linked keratin/PEO nanofibres mat; (b) first cross-linked keratin/PEO mat; (c) second cross-linked keratin/PEO mat.

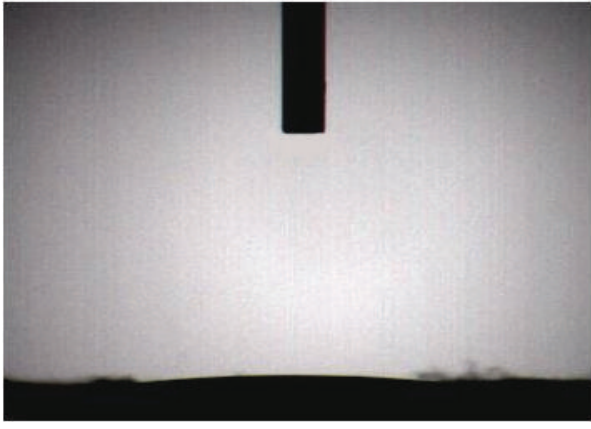

(a) First cross-linking keratin/PEO nanofibrous mat

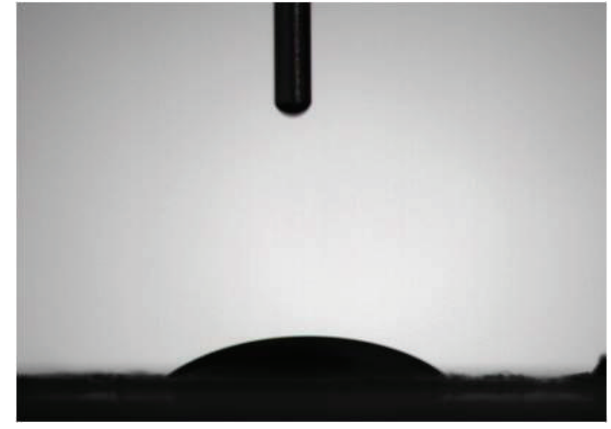

(b) Secondary cross-linking keratin/PEO nanofibrous mat

FIGURE 3: Contact angle images of (a) First cross-linked keratin/PEO nanofibers mat; (b) second cross-linked with EGDE vapor (99.5\%) keratin/PEO nanofibers mat.

reason might be that the low viscosity of spinning solution caused rise to break of jet [13]. Electrospun nanofibers with negligible bead defects were produced using the cross-linked spinning solution by EGDE (see Figure 2(b)), suggesting that EGDE could improve the spinnability of high content keratin solution. The improvement of fiber morphology is due to the cross-linking reaction between keratin molecules, which extends the keratin molecular length.

Figure 2(c) showed the morphology of the second crosslinked keratin/PEO nanofiber mat. Obviously, the morphologies of keratin/PEO nanofiber mat deteriorated slightly after the crosslinking. That may be due to the high temperature of the cross-link reaction with EGDE vapor.

3.2. Hydrophobicity of Nanofibers Mats. The water resistances of first cross-linked keratin/PEO nanofibers and secondary cross-linked keratin/PEO nanofibers were evaluated using a contact angle test apparatus. Contact angle images from the cross-linked keratin/PEO nanofibers are shown in Figure 3. For the first cross-linked keratin/PEO nanofibers, the angle was almost 0 (Figure 2(a)), suggesting that the first cross-linked keratin/PEO nanofibers are hydrophilic. 


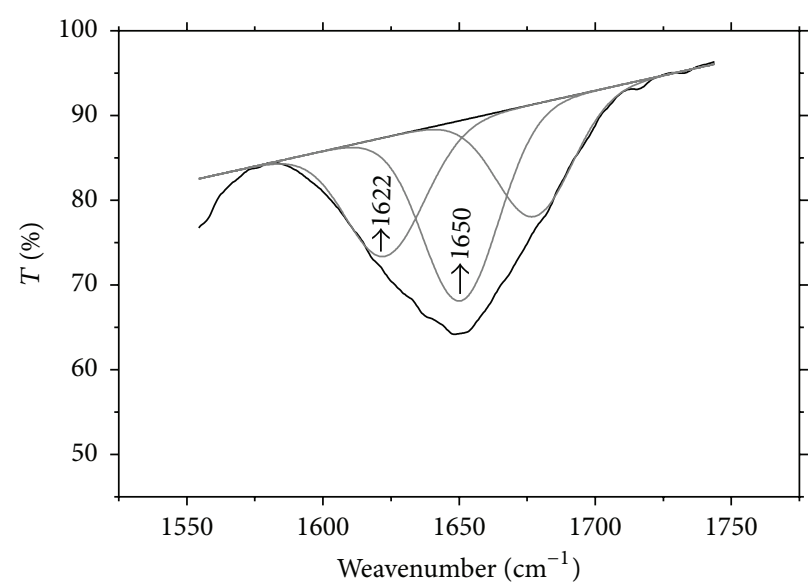

(a)

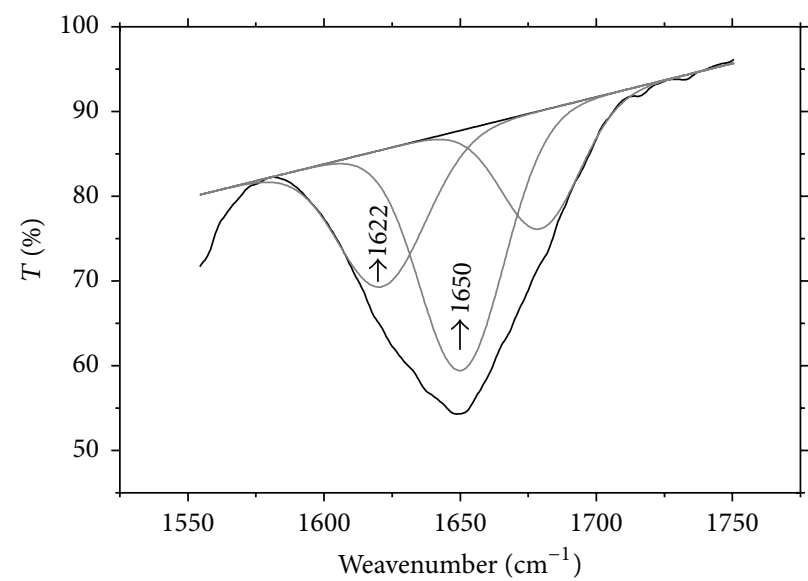

(b)

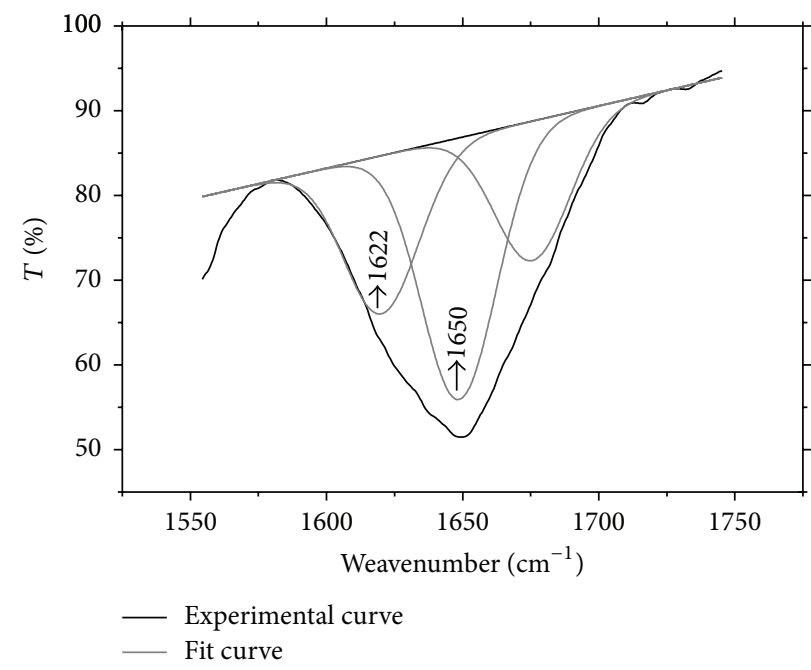

(c)

FIGURE 4: Curve-fitted amide I region of the keratin/PEO blend nanofiber mats.

The contribution of the first cross-linking reaction to the hydrophobicity of the fiber mat was negligible.

After secondary cross-linking with EGDE vapor, the contact angle of nanofiber mat increased to about $36.5^{\circ}$. The results showed that the secondary cross-linked method had a significant effect on the water resistances of keratin/PEO nanofibers.

3.3. FTIR Spectra Analysis. Infrared spectroscopy has been applied to investigate the molecular conformation of keratin/PEO nanofiber. The keratin component of the fiber gives the characteristic absorption bands in the regions named the amide A $\left(2800-3286 \mathrm{~cm}^{-1}\right)$, amide I $\left(1600-1700 \mathrm{~cm}^{-1}\right)$, amide II $\left(1480-1580 \mathrm{~cm}^{-1}\right)$, and amide III $\left(1220-1300 \mathrm{~cm}^{-1}\right)$ peaks, among which the amide I band corresponding to the $\mathrm{C}=\mathrm{O}$ stretching vibration is especially sensitive to the secondary structure of the proteins. And the amide I transmission band is not disturbed by the transmission peak generated by PEO and EGDE. As a result, the amide I band is appropriate to characterize the structural conformation of keratin in the blend nanofibers [13].

The secondary structure of keratin in the different nanofibers was compared by curve fitting of the amide I band. Firstly, the transmitted spectra were normalized at $3284 \mathrm{~cm}^{-1}$, at which the band corresponds to N-H stretching vibration of amide A. Since this transmission band was rarely affected by chemical treatment, it could be chosen as the band for spectrum normalization. Then, the amide I band was resolved in Gaussian-shaped bands at about $1622 \mathrm{~cm}^{-1}$ and $1650 \mathrm{~cm}^{-1}$ corresponding to the $\beta$-sheet structure and $\alpha$-helix structure of keratin, shown in Figure 4. The maximum peak height and content of the peak's corresponding structure were calculated from peak area, as listed in Table 1.

It can be seen from Figure 4 that the shapes of peaks at $1625 \mathrm{~cm}^{-1}$ and $1622 \mathrm{~cm}^{-1}$ are almost the same for the three nanofiber mats. Table 1 suggests that the $\beta$-sheet structure and $\alpha$-helix structure content of keratin almost do not change by the cross-link reaction by EGDE. The intensities of the two characteristic transmission peaks are gradually increased 
TABLE 1: Amide I absorption bands of keratin/PEO blend nanofiber mats.

\begin{tabular}{lcccccc}
\hline \multirow{2}{*}{ Wavenumber $\left(\mathrm{cm}^{-1}\right)$} & \multicolumn{2}{c}{ Uncross-linked nanofiber mat } & \multicolumn{2}{c}{ First cross-linked nanofiber mat } & \multicolumn{2}{c}{ Second cross-linked nanofiber mat } \\
& Max height & Content & Max height & Content & Max height & Content \\
\hline 1622 & 14.0 & 29.7 & 16.1 & 28.9 & 18.7 & 28.2 \\
1625 & 21.3 & 43.4 & 28.3 & 49.0 & 30.9 & 46.8 \\
\hline
\end{tabular}

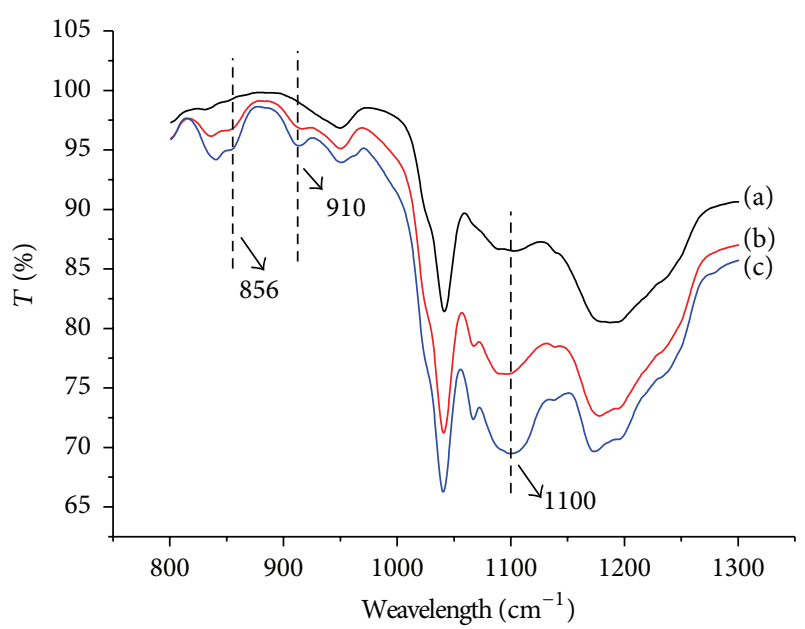

FIGURE 5: FTIR spectrum of keratin/PEO blend nanofiber mats (a) Untreated keratin/PEO nanofibers mat; (b) first cross-linking keratin/PEO nanofibrous mat; (c) secondary cross-linking keratin/PEO nanofibrous mat.

accompanying with the step-by-step cross-linking reaction (see Figure 4 and Table 1), meaning that the cross-linking reaction strengthened the absorption capability of keratin on its characteristic bands.

Previous research work showed that the characteristic transmission peak of $\mathrm{PEO}$ attributed to the C-O-C stretching vibration presents at $1094 \mathrm{~cm}^{-1}$ [21]. In addition, the peak value corresponding to the $\mathrm{C}-\mathrm{O}-\mathrm{C}$ stretching vibration would move to larger wave number at about $1102 \mathrm{~cm}^{-1}$ as the keratin content increased to $60 \%$ in the keratin/PEO blend materials. As a result, the weak peak at $1100 \mathrm{~cm}^{-1}$ of the uncross-linked nanofiber mat (line (a) in Figure 5) is due to the C-O-C stretching vibration of PEO for the three blend nanofiber mats with high keratin content [22]. The transmission spectrums of first and second cross-linked nanofiber mats in Figure 5 show that the $\mathrm{C}-\mathrm{O}-\mathrm{C}$ stretching vibration absorption are intensified by further cross-linking reaction with EGDE. That should be attributed to the epoxide groups in crosslinker EGDE.

The other two characteristic transmissions of EGDE located at the band $910 \mathrm{~cm}^{-1}$ and $859 \mathrm{~cm}^{-1}$ arise from the stretching vibrations of the epoxide groups in EGDE [23]. Figure 5 suggests that there are two weak transmission peaks at $910 \mathrm{~cm}^{-1}$ and $859 \mathrm{~cm}^{-1}$, which indicates that there are small amount of EGDE residual in the first and second cross-linked nanofiber mats.

3.4. Thermal Analysis. TG analysis was conducted to evaluate the effect of cross-linking treatment on keratin/PEO

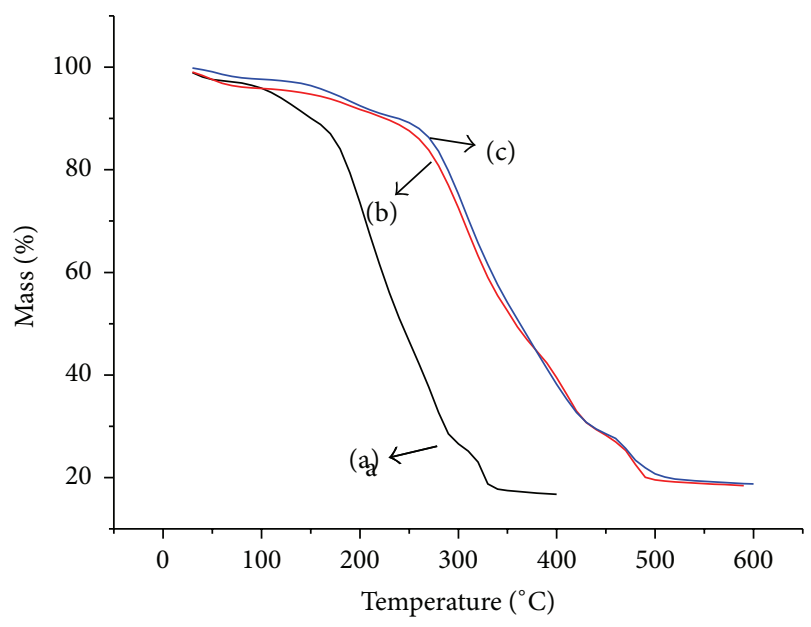

Figure 6: The TG curves of samples (a) untreated keratin/PEO nanofibers mat; (b) first cross-linking keratin/PEO nanofibrous mat; (c) secondary cross-linking keratin/PEO nanofibrous mat.

nanofiber mats. Figure 6 illustrates that the uncross-linked nanofiber mat presents lower thermal stability comparing with the first and second cross-linked nanofiber mats.

There are four weight loss stages of evident mass losses in thermogravimetric graphs (Table 2). The smallest percentage of weight loss in the first stage that occurred bellow $100^{\circ} \mathrm{C}$ is ascribed to the vaporization of the free water, physical adsorption of water, and the water of crystallization [24]. The second weight loss stage ranging from $127^{\circ} \mathrm{C}$ to $230^{\circ} \mathrm{C}$ in the TG curve of the first and second cross-linked fiber mats can be attributed to the melting of a-keratin [25]. The corresponding weight loss stage of the uncross-linked sample appeared in the range from $111^{\circ} \mathrm{C}$ to $154^{\circ} \mathrm{C}$ [26].

The third weight loss stage of the first and second crosslinked fiber mats emerge from $211^{\circ} \mathrm{C}$ to $384^{\circ} \mathrm{C}$ with the maximum decomposition rate at $307^{\circ} \mathrm{C}$, which corresponding to the decomposition of keratin [25]. The corresponding broad endothermic region of the uncross-linked sample emerges from $154^{\circ} \mathrm{C}$ to $258^{\circ} \mathrm{C}$ with the maximum decomposition rate at $209^{\circ} \mathrm{C}$ [22]. The last phase of mass loss is connected with the decomposition of keratin/PEO nanofibers. Weight loss analysis of nanofiber mats suggests that the lower temperature of denaturation/melting of a-keratin crystallites structure was shifted to the higher temperature after crosslinking treatment, suggesting that EGDE cross-link treatment can improve the thermal property of the keratin/PEO blend nanofiber mat.

3.5. X-Ray Diffraction Analysis. X-ray diffraction graphs were shown in Figure 7. It can be found that the typical 
TABLE 2: The weight loss and range of temperatures with different stages in the TG curves of different samples.

\begin{tabular}{|c|c|c|c|c|c|c|}
\hline & \multicolumn{2}{|c|}{ Untreated nanofiber mat } & \multicolumn{2}{|c|}{ First cross-linking nanofiber mat } & \multicolumn{2}{|c|}{ Second cross-linking nanofiber mat } \\
\hline & Temperature $\left({ }^{\circ} \mathrm{C}\right)$ & Weight loss (\%) & Temperature $\left({ }^{\circ} \mathrm{C}\right)$ & Weight loss (\%) & Temperature $\left({ }^{\circ} \mathrm{C}\right)$ & Weight loss (\%) \\
\hline Phase I & $20-111$ & 8.05 & $22-127$ & 3.98 & $36-127$ & 1.96 \\
\hline Phase II & $111-154$ & 21.01 & $127-211$ & 5.35 & $127-229$ & 7.15 \\
\hline Phase III & $154-258$ & 36.66 & $211-384$ & 36.77 & 229-384 & 49.6 \\
\hline Phase IV & $258-346$ & 12.18 & $384-445$ & 12.41 & $384-447$ & 12.30 \\
\hline
\end{tabular}

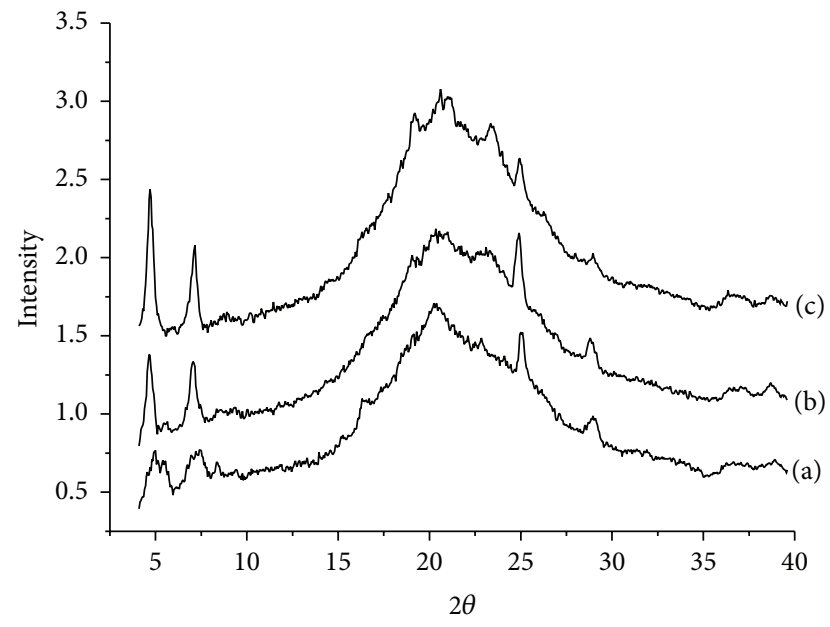

FIgURE 7: X-ray diffraction curves of the samples (a) untreated keratin/PEO nanofibers mat; (b) first cross-linking keratin/PEO nanofibrous mat; (c) secondary cross-linking keratin/PEO nanofibrous mat.

diffraction pattern of keratin located at $2 \theta=10^{\circ}$ almost disappeared in the keratin/PEO blend nanofiber mats [26, 27]. Meanwhile, two diffraction peaks at $2 \theta=4.7^{\circ}$ and $7.0^{\circ}$ emerge in the diffraction curve of the uncross-linked keratin/PEO blend nanofiber mats, and the intensity of the two peaks gradually intensified by the cross-linking reaction with EGDE. That means the emergency of the two peaks have a close relationship with the chemical reaction of keratin with the cross-linker.

The crystallinities of the three samples were calculated based on the X-ray diffraction curves. The crystalline and amorphous profiles could be obtained by curve decomposition using Gaussian function, as shown in Figure 8. The degree of crystallinity was determined based on the estimation ratio of the crystalline to amorphous material in the sample and listed in Table 3.

The results showed that the crystallinity of the keratin/PEO blend nanofiber mat increased from $13.14 \%$ for the uncross-linked sample to $21.54 \%$ and $35.15 \%$ for the first cross-linked and second cross-linked samples, respectively. The reason might be attributed to a new crystalline structure from the chemical reaction between cyclic ether bond of EGDE and the hydroxyl group of the keratin. In addition, the increased crystallinity of the first cross-linked and second cross-linked nanofiber mats keeps well with their improved thermal stability by TG test.

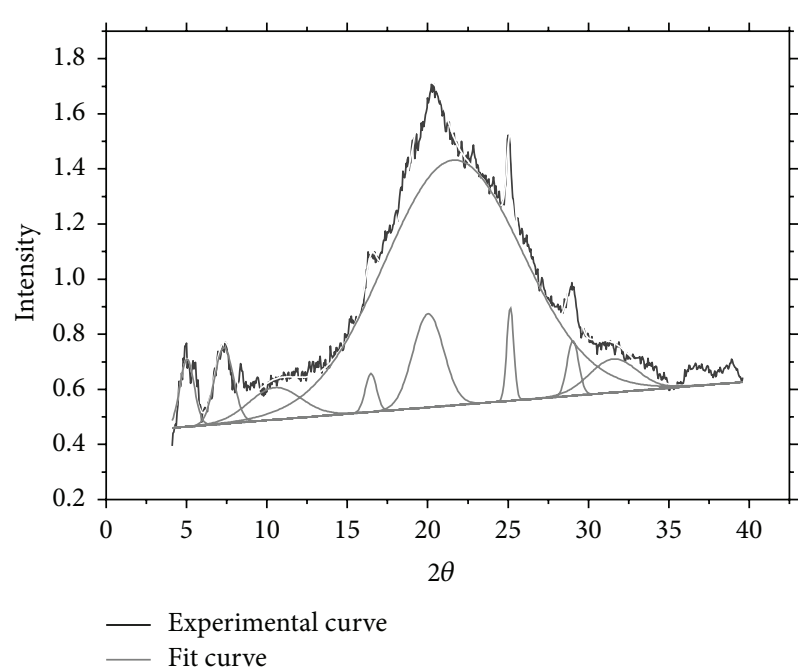

FIGURE 8: Curve decomposition of the uncross-linked keratin/PEO blend nanofiber mats.

TABLE 3: Crystallinity of the keratin/PEO blend mats.

\begin{tabular}{lc}
\hline Sample & Crystallinity \\
\hline Uncross-linked & 13.14 \\
First cross-linked & 21.54 \\
Secondary cross-linked & 35.15 \\
\hline
\end{tabular}

\section{Conclusions}

Keratin was extracted from human hair, and blended with PEO in aqueous solutions. The EGDE, as cross-linker, was firstly added to the high-content keratin/PEO (90/10) aqueous solutions for preparing nanofibers with negligible bead defects. EGDE vapor were then employed to cross-link keratin/PEO (90/10) nanofibers. The results showed that the use of cross-linker is capable of improving electrospinnability of keratin and could produce high-content keratin/PEO nanofibers mat. The secondary cross-linking through EGDE vapor could increase the hydrophobic property and the crystallinity of keratin/PEO (90/10) nanofibers mat. This study opens an effective way to fabricate the high-content keratin nanofibrous mats for cells growth or tissue engineering.

\section{Conflict of Interests}

There is no conflict of interests regarding the publication of this paper. 


\section{Acknowledgments}

The present work is supported by National Natural Science Foundation of China under Grant (no. 51003073), Foundation for the Author of National Excellent Doctoral Dissertation of PR China (no. 201255), Program for New Century Excellent Talents in University (NCET-12-1063), Tianjin Natural Science Foundation (14JCYBJC17600), Ningbo Natural Science Foundation (2013A610016), and China Postdoctoral Science Foundation Grants nos. 2012M521122 and 2013 T60559.

\section{References}

[1] L. Michaelis, "A study of keratin," Journal of the American Leather Chemists Association, vol. 30, no. 11, pp. 557-568, 1935.

[2] K. Yamauchi, A. Yamauchi, T. Kusunoki, A. Kohda, and Y. Konishi, "Preparation of stable aqueous solution of keratins, and physiochemical and biodegradational properties of films," Journal of Biomedical Materials Research, vol. 31, no. 4, pp. 439444, 1996.

[3] K. Yamauchi and A. Khoda, "Novel proteinous microcapsules from wool keratins," Colloids and Surfaces B, vol. 9, pp. 117-119, 1997.

[4] A. Tachibana, Y. Furuta, H. Takeshima, T. Tanabe, and K. Yamauchi, "Fabrication of wool keratin sponge scaffolds for long-term cell cultivation," Journal of Biotechnology, vol. 93, no. 2, pp. 165-170, 2002.

[5] T. Tanabe, N. Okitsu, A. Tachibana, and K. Yamauchi, "Preparation and characterization of keratin-chitosan composite film," Biomaterials, vol. 23, no. 3, pp. 817-825, 2002.

[6] K. Katoh, M. Shibayama, T. Tanabe, and K. Yamauchi, "Preparation and physicochemical properties of compression-molded keratin films," Biomaterials, vol. 25, no. 12, pp. 2265-2272, 2004.

[7] K. Katoh, T. Tanabe, and K. Yamauchi, "Novel approach to fabricate keratin sponge scaffolds with controlled pore size and porosity," Biomaterials, vol. 25, no. 18, pp. 4255-4262, 2004.

[8] W. T. Sow, Y. S. Lui, and K. W. Ng, "Electrospun human keratin matrices as templates for tissue regeneration," Nanomedicine, vol. 8, no. 4, pp. 531-541, 2013.

[9] D. B. Khadka and D. T. Haynie, "Protein- and peptide-based electrospun nanofibers in medical biomaterials," Nanomedicine: Nanotechnology, Biology, and Medicine, vol. 8, no. 8, pp. 12421262, 2012.

[10] Y. Liu, J. Li, Y. Tian, J. Liu, and J. Fan, "Multi-physics coupled fem method to simulate the formation of crater-like taylor cone in electrospinning of nanofibers," Journal of Nano Research, vol. 27, pp. 153-162, 2014.

[11] Y. Liu, J. Li, Y. Tian, X. Yu, J. Liu, and B.-M. Zhou, "CLSVOF method to study the formation process of Taylor cone in craterlike electrospinning of nanofibers," Journal of Nanomaterials, vol. 2014, Article ID 635609, 12 pages, 2014.

[12] J.-H. He, Y. Liu, L.-F. Mo, Y.-Q. Wan, and L. Xu, Electrospun Nanofibres and Their Applications, Smithers Rapra Technology, Shropshire, UK, 2008.

[13] A. Aluigi, C. Vineis, A. Varesano, G. Mazzuchetti, F. Ferrero, and C. Tonin, "Structure and properties of keratin/PEO blend nanofibres," European Polymer Journal, vol. 44, no. 8, pp. 24652475, 2008.

[14] J. Li, Y. Li, L. Li, A. F. T. Mak, F. Ko, and L. Qin, "Preparation and biodegradation of electrospun PLLA/keratin nonwoven fibrous membrane," Polymer Degradation and Stability, vol. 94, no. 10, pp. 1800-1807, 2009.

[15] H. Thomas, E. Heine, R. Wollseifen, C. Cimpeanu, and M. Möller, "Nanofibers from natural and inorganic polymers via electrospinning," International Nonwovens, vol. 14, no. 3, pp. 12 $18,2005$.

[16] J. Choi, G. Panthi, Y. Liu et al., "Keratin/poly (vinyl alcohol) blended nanofibers with high optical transmittance," Polymer, vol. 58, pp. 146-152, 2015.

[17] J. G. Rouse and M. E. van Dyke, "A review of keratin-based biomaterials for biomedical applications," Materials, vol. 3, no. 2, pp. 999-1014, 2010.

[18] A. Varesano, A. Aluigi, C. Vineis, and C. Tonin, "Study on the shear viscosity behavior of keratin/PEO blends for nanofibre electrospinning," Journal of Polymer Science. Part B: Polymer Physics, vol. 46, no. 12, pp. 1193-1201, 2008.

[19] Z.-C. Xin, J. Yuan, W.-P. Chae, and I.-K. Kang, "Keratin nanofibers as a biomaterial," in 2010 International Conference on Nanotechnology and Biosensors, vol. 2, pp. 120-124, IACSIT Press, Singapore, 2011.

[20] A. Aluigi, A. Varesano, A. Montarsolo et al., "Electrospinning of keratin/poly(ethylene oxide) blend nanofibers," Journal of Applied Polymer Science, vol. 104, no. 2, pp. 863-870, 2007.

[21] X. Li and S. L. Hsu, "An analysis of the crystallization behavior of poly(ethylene oxide)/poly(methyl methacrylate) blends by spectroscopic and calorimetric techniques," Journal of Polymer Science: Polymer Physics Edition, vol. 22, no. 7, pp. 1331-1342, 1984.

[22] C. Tonin, A. Aluigi, C. Vineis, A. Varesano, A. Montarsolo, and F. Ferrero, "Thermal and structural characterization of poly(ethylene-oxide)/keratin blend films," Journal of Thermal Analysis and Calorimetry, vol. 89, no. 2, pp. 601-608, 2007.

[23] D. Huang, Z. Peng, Z. Hu et al., "A new consolidation system for aged silk fabrics: effect of reactive epoxide-ethylene glycol diglycidyl ether," Reactive and Functional Polymers, vol. 73, no. 1, pp. 168-174, 2013.

[24] Z. Éhen, C. Novák, J. Sztatisz, and O. Bene, “Thermal characterization of hair using TG-MS combined thermoanalytical technique," Journal of Thermal Analysis and Calorimetry, vol. 78, no. 2, pp. 427-440, 2004.

[25] N. Eslahi, F. Dadashian, and N. H. Nejad, "Optimization of enzymatic hydrolysis of wool fibers for nanoparticles production using response surface methodology," Advanced Powder Technology, vol. 24, no. 1, pp. 416-426, 2013.

[26] J. Cao, "Melting study of the $\alpha$-form crystallites in human hair keratin by DSC," Thermochimica Acta, vol. 335, no. 1-2, pp. 5-9, 1999.

[27] J. Cao and C. A. Billows, "Crystallinity determination of native and stretched wool by X-ray diffraction," Polymer International, vol. 48, no. 10, pp. 1027-1033, 1999. 

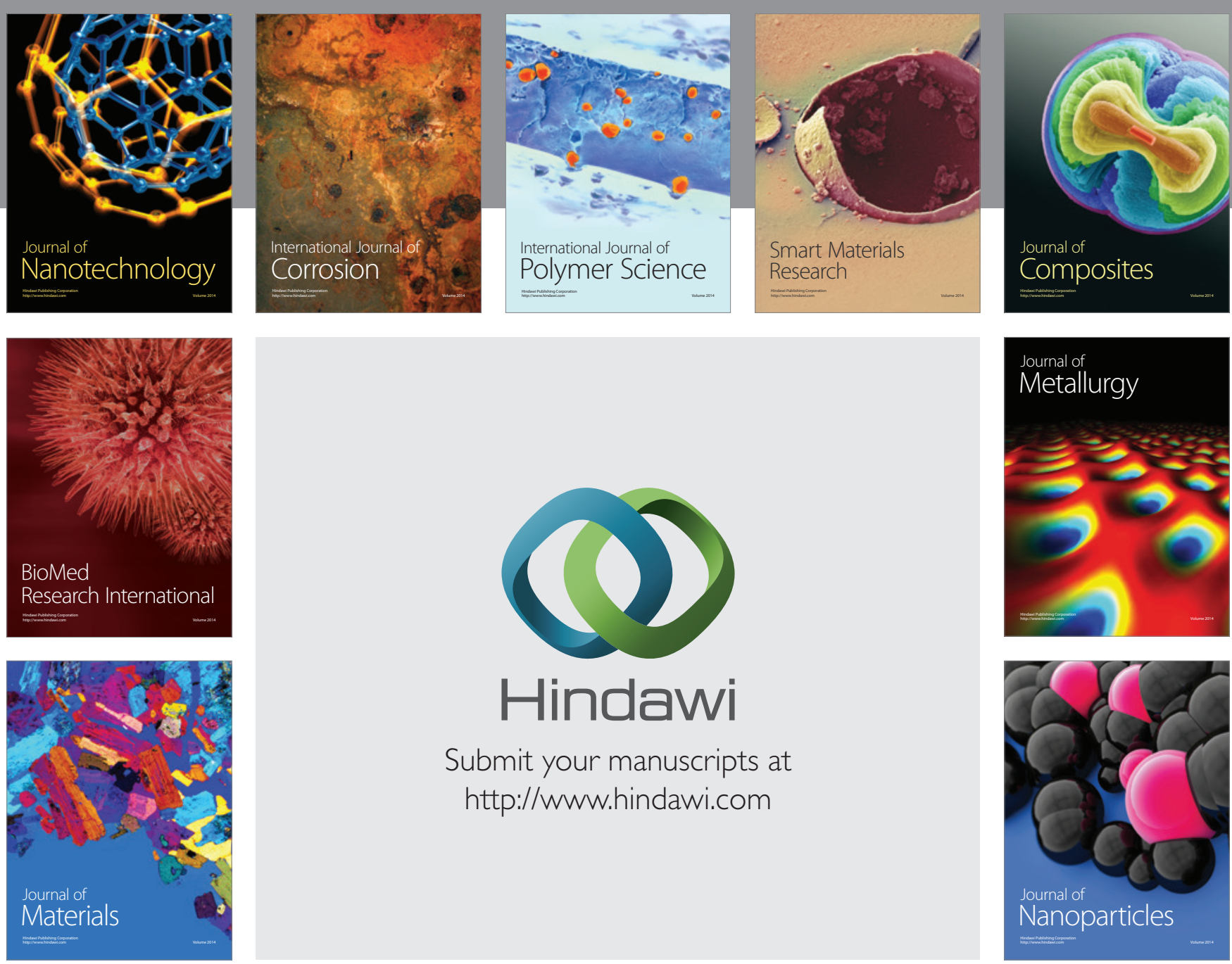

Submit your manuscripts at http://www.hindawi.com
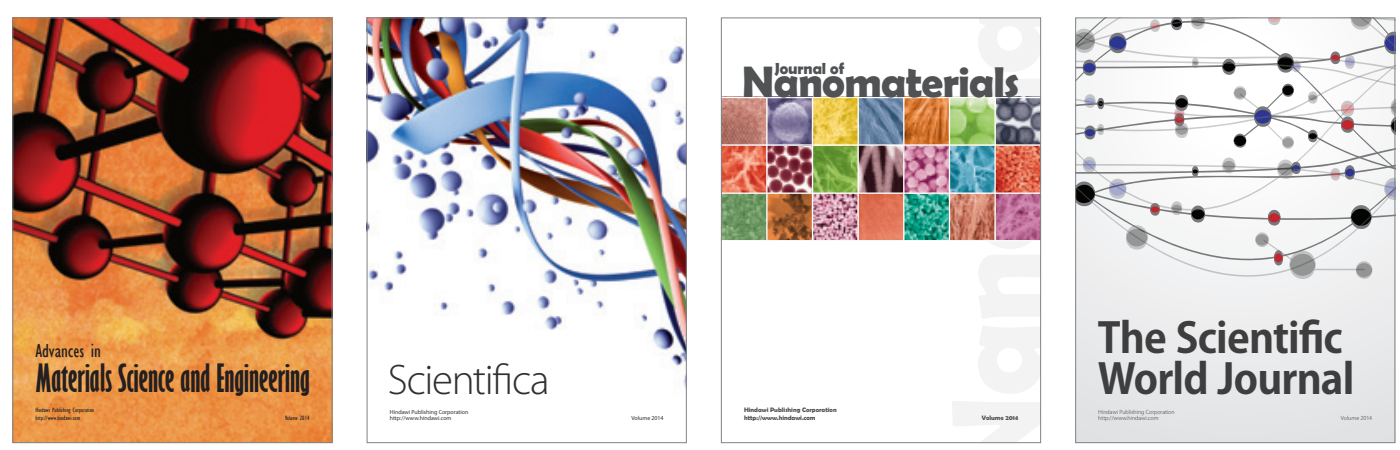

\section{The Scientific World Journal}
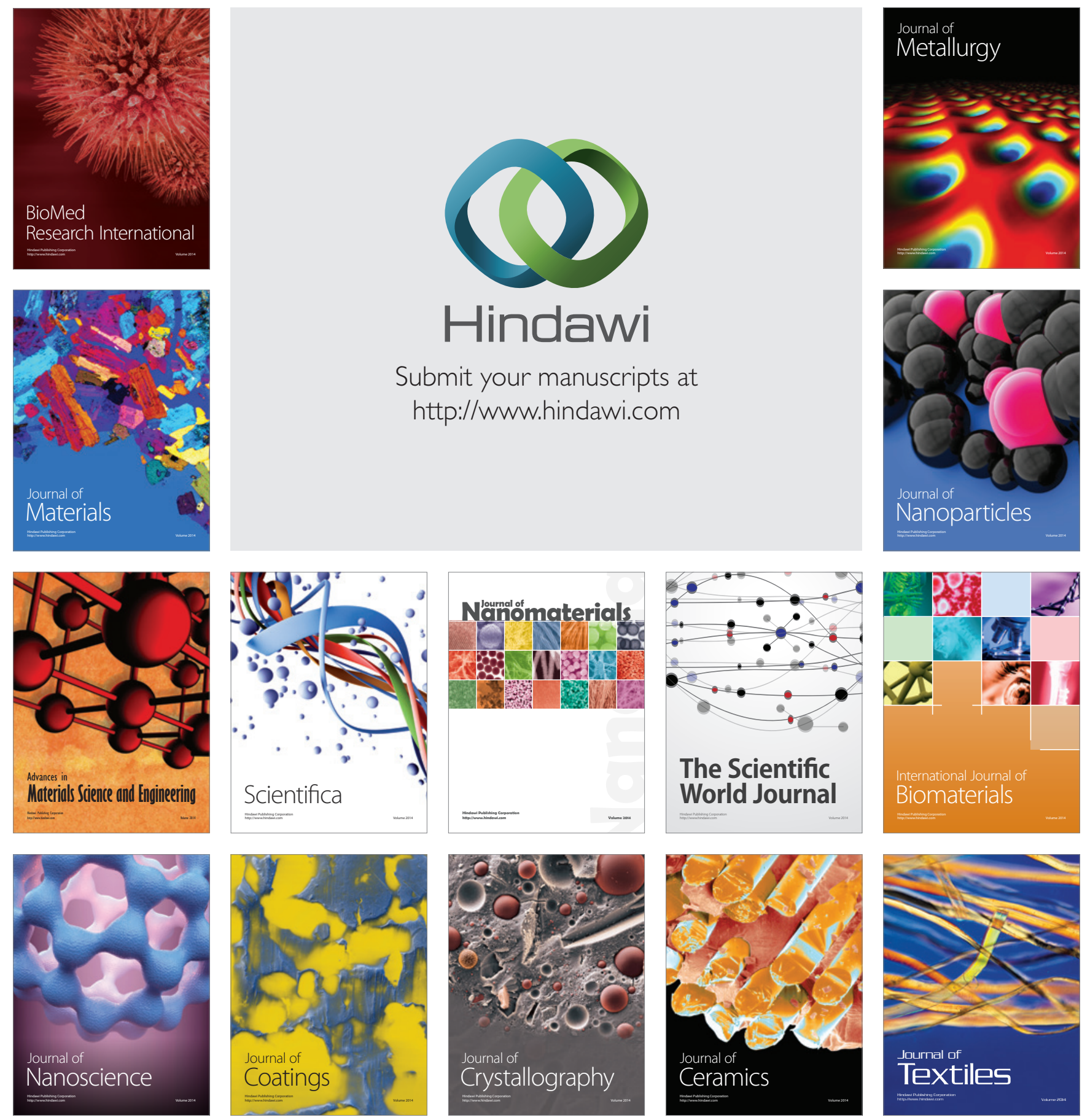